\title{
ANESTÉSICOS LOCAIS EM ODONTOLOGIA: UMA REVISÃO DE LITERATURA
}

\section{LOCAL ANESTHETICS IN DENTISTRY: A LITERATURE REVIEW}

\author{
Leonardo Costa de Almeida Paiva ${ }^{1}$, Alessandro Leite Cavalcanti ${ }^{2}$ \\ 1 Universidade Estadual da Paraíba - UEPB, Curso de Odontologia, Campina Grande, PB, \\ Brasil; (83) 3321-2971; e-mail: leonardocap@terra.com.br \\ 2 Universidade Estadual da Paraíba - UEPB, Departamento de Odontologia, \\ Campina Grande, PB
}

Recebido para publicação em 24/10/2005

Aceito para publicação em 03/11/2005

\section{RESUMO}

A farmacologia dos anestésicos locais é complexa e novas drogas surgem diariamente. Logo, a compreensão dos aspectos farmacológicos dos anestésicos locais é importante para a seleção da droga a ser utilizada em cirurgia. As propriedades físico-químicas de cada anestésico local determinam a ação, potencialidade e duração da anestesia. Como orientação ao clínico-geral, este artigo fornece informações importantes sobre os anestésicos locais.

Palavras-chave: anestesia dentária, anestésicos locais, farmacologia

\begin{abstract}
The pharmacology of local anesthetics is complex, and new drugs emerge every day. The understanding of the pharmacology of local anesthesia is important for the selection of a local anesthetic for use in surgery. The physicochemical properties of each local anesthetic determine the onset of action, potency, and duration. Clinically oriented, this paper provides detailed information for those practitioners who use local anesthetics.
\end{abstract}

Key words: dental anesthesia, local anesthetics, pharmacology 


\section{Introdução}

A anestesia local é definida como um bloqueio reversível da condução nervosa, determinando perda das sensações sem alteração do nível de consciência (Ferreira, 1999).

A procura por substâncias que pudessem amenizar a sensação dolorosa vem desde a antiguidade, onde já se conhecia o ópio (suco da papoula). Antes da descoberta dos anestésicos, também eram utilizados, asfixia temporária do paciente na qual se provocava uma isquemia cerebral e um desmaio momentâneo (se necessário dava-se uma pancada na cabeça do paciente, atordoando-o). Nessa última opção, caso este procedimento não resolvesse, o paciente era vigorosamente imobilizado pelos membros por quatro auxiliares e o cirurgião poderia realizar o seu esperado trabalho (Faria; Marzola, 2001).

Nieman, em 1860, utilizou o primeiro anestésico local na Medicina e Odontologia que foi a cocaína, isolada da Erytroxycolon coca. No ano de 1880, Von Srep desenvolveu um estudo de suas propriedades farmacológicas. Os benefícios da cocaína foram bastante apreciados e logo passou a ser administrada com eficácia em vários procedimentos médicos e odontológicos. Várias pesquisas tiveram início à procura de substitutos sintéticos para a cocaína, tendo Ein Horn, em 1905, sintetizado a procaína, que deu início à descoberta dos anestésicos locais utilizados até hoje (Tortamano; Armonia, 2003).

Atualmente, os anestésicos locais mais utilizados em Odontologia são aminas terciárias com propriedades hidrofílicas e lipofílicas, sintetizados na década de 40. Constituem-se em uma alternativa menos tóxica, mais efetiva e com potencial alergênico menor que os anestésicos tipo éster. (Vieira; Gonçalves; Agra, 2000).

Este trabalho tem por objetivo, por meio de uma revisão de literatura, tecer considerações sobre os anestésicos locais, enfocando os aspectos relativos à farmacologia, às principais reações adversas, ao uso dos vasoconstrictores, às indicações e contra-indicações e o uso durante a gravidez.

\section{Farmacologia}

Pode-se dividir a molécula do anestésico local em três partes: um grupamento amínico secundário ou terciário que confere às moléculas hidrossolubilidade. Outro, aromático que concede às moléculas propriedades lipofílicas que são essenciais para a sua penetração nas fibras nervosas. Por último, unindo essas duas partes, uma cadeia intermediária que é importante em dois aspectos. Primeiramente, fornece a separação espacial necessária entre as extremidades lipofílica e hidrofílica e também a ligação química entre os dois grupamentos, servindo como base para a classificação dos anestésicos locais em dois grupos: os ésteres (-COO) e as amidas (-NHCO-). A cadeia intermediária é de grande relevância, já que há grandes diferenças no grau de alergenicidade, na potência e no metabolismo, quando comparamos os dois grupos de fármacos (Tortamano; Armonia, 2001).

A anestesia local determina abolição de funções autonômicas e sensitivomotoras. O comprometimento da condução em fibras periféricas obedece à determinada seqüência, em que primeiramente se bloqueiam as autonômicas, depois as responsáveis pelas sensibilidades térmica, dolorosa e tátil, a seguir as relacionadas à pressão e vibração e por último, as proprioceptivas e motoras. Essa seqüência depende do diâmetro, porção e da mielinização das fibras nervosas. A recuperação das funções nervosas se dá na ordem inversa (Ferreira, 1999).

Um bom agente anestésico deve apresentar baixa toxicidade sistêmica; não ser irritante aos tecidos e também não causar lesão permanente às estruturas nervosas. O tempo para início da anestesia deve ser o mais curto possível e a duração de ação suficiente para a realização do procedimento cirúrgico, com ação reversível (Faria; Marzola, 2001; Malamed, 2004).

De acordo com Tortamano e Armonia (2001), os anestésicos locais além de realizarem o bloqueio da condução nervosa, também interferem na função de todos os órgãos nos quais ocorrem condução ou transmissão de impulsos nervosos. Assim sendo, exercem ação sobre o sistema nervoso central (SNC), gânglios autonômicos, função neuromuscular e em todos os tipos de fibras musculares. No SNC, o estímulo é seguido de depressão idêntica à causada pelos anestésicos gerais, nos quais doses extremamente altas prejudicam a função respiratória, podendo levar a óbito por asfixia.

A duração da anestesia é determinada pelo grau de ligação protéica. Os anestésicos que apresentam 
grande afinidade ao componente protéico do nervo têm menos probabilidade de se difundirem do local da injeção e serem absorvidos pela circulação sistêmica (Ranali; Volpato, 1990). Apesar dessas drogas serem bases fracas, as preparações farmacêuticas (sais de hidrocloreto) são levemente ácidas, com $\mathrm{pH}$ variando de 4,5 a 6,0 nos tubetes odontológicos, sendo que esta acidez aumenta a estabilidade das soluções anestésicas. Uma vez injetados nos tecidos, com $\mathrm{pH}$ mais alcalino ( $\mathrm{pH}=7,4)$, há tamponamento ácido, liberando base em forma não-ionizada, passível de ser absorvida. Devido à instabilidade e reduzida solubilidade, quando em solução, são comercializados na forma de sais hidrossolúveis, geralmente cloridratos (Ferreira, 1999; Tortamano; Armonia, 2001).

\section{Farmacocinética}

É de relevância no que se refere à absorção, que essas substâncias, quando injetadas nos tecidos moles exerçam uma ação farmacológica nos vasos sanguíneos da área. Todos apresentam algum grau de vasoatividade, sendo na maioria vasodilatadoras. A exceção é a cocaína, que também é a única substância que tem uma significativa absorção pelo trato gastrointestinal, quando administrada por via oral.

Após absorvidos pela corrente sanguínea, os anestésicos locais são distribuídos para todos os tecidos do corpo, apresentando uma meia-vida que vai de alguns minutos a algumas horas, dependendo da droga empregada. Os órgãos e áreas altamente perfundidos, como o cérebro, fígado, rins, pulmões e baço, apresentam inicialmente maiores níveis sanguíneos do anestésico do que aqueles menos perfundidos.

Quanto à biotransformação há uma relativa diferença entre os anestésicos do tipo éster e os do tipo amida. Enquanto os primeiros são hidrolisados por colinesterases plasmáticas, os segundos por enzimas microssomais hepáticas, tendo uma maior duração de efeito que aqueles. Faz-se exceção a tetracaína (tipo éster), cujo efeito é mais prolongado. O metabolismo do anestésico local é importante, pois a toxicidade geral da droga depende do equilíbrio entre a velocidade de absorção para a corrente sanguínea no local da injeção e a velocidade em que ela é removida do sangue, através dos processos de absorção tecidual e metabolismo.

No tocante à excreção, os rins são os órgãos excretores primários tanto para os anestésicos locais quanto para seus metabólitos. Doenças renais significativas representam uma contra-indicação relativa à administração de anestésicos locais, já que os rins podem ser incapazes de eliminar do sangue o anestésico original ou seus principais metabólitos, resultando em um ligeiro aumento dos níveis sanguíneos desse composto e um aumento no potencial de toxicidade (Veering, 2003; Malamed, 2004; Tortamano; Armonia, 2001).

\section{Anestésicos locais mais utilizados em Odontologia}

Dentre os anestésicos locais comercializados, os mais utilizados na Odontologia são a lidocaína, a prilocaína, a mepivacaína e a bupivacaína. Incluem-se ainda a articaína, a ropivacaína e a levobupivacaína, sendo estas duas últimas, alternativas mais seguras para a bupivacaína, por apresentar menos toxicidade sistêmica (Veering, 2003).

\section{Lidocaína}

A lidocaína é considerada o anestésico padrão em Odontologia, com o qual todos os outros anestésicos são comparados. Foi o primeiro agente anestésico do grupo amida a ser sintetizado, em 1943 por Nils Lofgren. Inicia sua ação por volta de 2 a 3 minutos e tem eficácia em uma concentração de $2 \%$. Sua dose máxima recomendada é de 7,0mg/Kg em adultos, não excedendo 500mg ou 13 tubetes anestésicos. É encontrada comercialmente nas concentrações de $1 \%$ e $2 \%$, com ou sem vasoconstrictor. Para aplicação tópica sua concentração pode ser de 5\% (Vieira; Gonçalvez; Agra, 2000; Mariano; Santana; Coura, 2000; Malamed, 2004; DEF, 2004).

\section{Prilocaína}

Malamed(2004) reportou que foi sintetizada pela primeira vez em 1953 por Lofgren e Tegnér, tendo sido descrita apenas em 1960. Apresenta uma potência e toxicidade duas vezes maior que a lidocaína e um início de ação mais retardado, por volta de 2 a 4 minutos. A dose máxima recomendada é de $6,0 \mathrm{mg} / \mathrm{kg}$, não excedendo $400 \mathrm{mg}$ ou 7 tubetes anestésicos na concentração de 4\%, no paciente adulto. A concentração odontológica eficaz é de 4\%. Esse anestésico não apresenta formulação tópica. A técnica infiltrativa 
oferece pouco tempo de anestesia pulpar, enquanto o bloqueio regional fornece uma anestesia pulpar de até 60 minutos. Comercialmente, só é encontrado na concentração 3\% e tendo a felipressina como vasoconstrictor (DEF, 2004). Formulações genéricas podem ser encontradas na concentração de $4 \%$, sendo estas as que provêm melhores resultados.

\section{Mepivacaína}

É amplamente utilizada no campo odontológico, sendo classificada como um anestésico de duração intermediária. Foi preparada por A. F. Ekenstam, em 1957 e introduzida na odontologia em 1960. Apresenta potência e toxicidade duas vezes maior que a lidocaína, tendo o seu início da ação por volta de 1 1 1/2 a 2 minutos. A dose máxima é de 6,6 mg/kg, não devendo ultrapassar 400mg ou 11 tubetes anestésicos. A concentração odontológica eficaz é de $2 \%$ (com vasoconstritor) e de $3 \%$ (sem vasoconstritor). Uma de suas vantagens é que esta substância consegue ter um tempo maior de anestesia do que os outros anestésicos sem o uso do vasoconstrictor. É sintetizada apenas por laboratórios especializados em artigos odontológicos (Ponzoni; Sanches; Okamoto, 2003; Malamed, 2004; DEF, 2004).

\section{Cloridrato de Bupivacaína}

No Brasil, dentre os anestésicos de longa duração, somente o cloridrato de bupivacaína está disponível comercialmente. Apresenta potência quatro vezes maior que a lidocaína e uma toxicidade quatro vezes menor. Inicia sua ação por volta de 6 a 10 minutos. Apresenta uma dose máxima recomendada de 1,3mg/ kg, não devendo ultrapassar 90mg ou 10 tubetes.

Quanto ao tempo de duração, a anestesia mandibular pode persistir de 5 a 9 horas. Os estudos sobre sua toxicidade mostram que ocorrem devido à superdosagem ou por injeção acidental do anestésico nos vasos sanguíneos, não sendo essas reações diferentes das que ocorrem com os outros anestésicos locais. Em tubetes anestésicos é encontrado na concentração de 0,5\%, porém em ampolas de $20 \mathrm{ml}$ podem ser encontrados nas concentrações de 0,25\%, 0,50\% e 0,75\% (com ou sem vasoconstritor). É o anestésico mais utilizado em recintos hospitalares (DEF, 2004; Ranali; Volpato, 1990; Malamed, 2004).

\section{Articaína}

A articaína foi aprovada para uso nos Estados Unidos em abril de 2000, tendo como nome comercial Steptocaine 4\% com 1:100.000 de epinefrina. Sua dose máxima recomendada é de $6,6 \mathrm{mg} / \mathrm{kg}$, não ultrapassando 500mg ou 6 tubetes (Mikesell et al., 2005; DEF, 2004).

\section{Vasoconstritores}

Os vasoconstritores são importantes componentes das soluções anestésicas. No passado, atribuíamse várias desvantagens a eles, porém muitas delas decorriam em função do uso inadequado: injeções intravasculares, concentrações elevadas, aplicações rápidas e grandes volumes, levando à intoxicação relativa. Hoje, sabe-se que quase nenhuma solução anestésica teria efeito sem o emprego dos vasoconstrictores, tendo como principal vantagem a absorção lenta do sal anestésico, que reduz a toxicidade deste, aumenta a duração da anestesia, possibilita o uso de quantidades menores de solução, além de aumentar o efeito anestésico (Mariano; Santana; Coura, 2000).

As substâncias vasoconstritoras podem pertencer a dois grupos farmacológicos: aminas simpatomiméticas e análogos da vasopressina. As mais comuns são a adrenalina/epinefrina, a noradrenalina/noraepinefrina, a fenilefrina e o octapressin/ felipressina.

A adrenalina é também uma substância endógena, produzida pelas supra-renais quando o SNC é ativado. Essa substância tem a capacidade de se ligar aos receptores e $\alpha$ e $\beta$ dos órgãos inervados pelo simpático e de produzir a célebre "reação de alarme", descrita por Cânon, na década de 40, que prepara o animal para a luta ou fuga (Faria; Marzola, 2001; Ferreira, 1999).

Normalmente, os vasoconstritores associados aos anestésicos locais não produzem efeitos farmacológicos, além da constrição arteriolar localizada. Uma das maiores polêmicas que existe em relação aos vasoconstritores, é o de usá-los ou não em pacientes cardiopatas, uma vez que a adrenalina eleva a pressão sistólica e a freqüência cardíaca, causando palpitações e dor torácica. A felinefrina não provoca estímulo cardíaco direto, mas pode elevar de forma significativa as pres- 
sões sistólica e diastólica. Como conseqüência destas alterações, através do reflexo vagal, provoca-se a queda da freqüência cardíaca.

A noradrenalina eleva as pressões sistólica e diastólica e praticamente não interfere com a freqüência cardíaca. Por induzir vasoconstrição mais acentuada, o dano tecidual é maior, o que leva alguns autores desaconselharem o uso deste vasoconstrictor.

A felipressina ou octapressin, é uma análoga sintética da vasopressina, hormônio produzido pela neurohipófise e também conhecida como hormônio antidiurético ou $\mathrm{ADH}$. Não apresenta efeitos diretos sobre o miocárdio, mas é um potente vasoconstritor coronariano o que pode levar a crises de angina com isquemia miocárdica, em pacientes com alguma deficiência na circulação coronariana (Faria; Marzola, 2001; Ferreira, 1999; Tortamano; Armonia, 2001).

Segundo Faria e Marzola (2001), caso aconteça alguma complicação com o paciente não será pelo vasoconstrictor do anestésico, mas sim pelas catecolaminas endógenas liberadas na circulação, já que a quantidade liberada, em uma situação de estresse, é muito acima da contida em um tubete odontológico, tornandose irrisória a quantidade ali presente.

Como contra-indicações no uso de vasocontritores, a literatura cita angina pectóris instável, infarto do miocárdio recente (até 6 meses), acidente vascular cerebral recente, cirurgia de revascularização miocárdica recente, arritmias refratárias, insuficiência cardíaca congestiva intratável ou não-controlada, hipertensão grave não-tratada ou não-controlada, hipertireoidismo não-controlado, diabete mellitus não-controlado, feocromocitoma e hipersensibilidade a sulfitos (Ferreira, 1999; Mariano; Santana; Coura, 2000; De Castro et al., 2002).

\section{Reações Adversas}

As complicações quando ocorrem, podem ser divididas em psicogênicas e não-psicogênicas. As primeiras independem do anestésico e estão relacionadas ao estado de estresse do paciente. As ocorrências mais comuns são a lipotímia e a hiperventilação. As não-psicogênicas são raras, estando relacionadas à técnica de administração inadequada, superdosagem ou a uma reação alérgica ao anestésico(Vieira; Gonçalves; Agra, 2000).
Grande parte dos eventos considerados alérgicos decorre de reações tóxicas aos anestésicos locais diretamente no SNC e cardiovascular. Os anestésicos locais podem desencadear reações alérgicas dos tipos I (hipersensibilidade imediata) e IV (dermatite de contato). Os do tipo éster causam reações do tipo IV, enquanto os do tipo amida podem causar ambos os tipos de hipersensibilidade.

As manifestações clínicas sugestivas de hipersensibilidade mediada por IgE incluem prurido, urticária, broncoespasmo e angioedema. Na maioria dos casos, esses eventos ocorrem até uma hora após a exposição. Outras ocorrências tais como dispnéia, hipertensão arterial ou síncope, poderiam ser eventualmente mediadas por IgE, entretanto, podem envolver outros mecanismos. Deve-se ter cuidado com os pacientes asmáticos alérgicos, principalmente, os dependente de corticósteróides, pois geralmente apresentam alergia aos sulfitos encontrados nas soluções contendo aminas simpatomiméticas, sendo nesse caso indicado soluções com felipressina (Araújo; Amaral, 2004; Berkun et al., 2003; Mariano; Santana; Coura, 2000).

Uma enfermidade, que pode acometer o paciente quando do uso dos anestésicos locais e que os cirurgiões-dentistas não estão habituados a lidar a observar como rotina, é a metahemoglobinemia. Trata-se de uma cianose que ocorre na ausência de anormalidades cardíacas e/ou respiratórias, podendo ser congênita ou adquirida. Os anestésicos que mais causam a metahemoglobinemia são a prilocaína, a articaína e a benzocaína (uso tópico), os quais devem ser evitados em grandes cirurgias, portadores de insuficiência cardíaca, respiratória ou doenças metabólicas e em gestantes, por causa do risco do feto vir a contrair a doença. $\mathrm{O}$ paciente se apresenta letárgico, com os leitos ungueais e as mucosas cianóticas, dificuldades respiratórias e a pele em tom cinza pálido. O seu tratamento se dá através da administração intravenosa de azul-de-metileno a $1 \%(1,5 \mathrm{mg} / \mathrm{Kg})$, podendo a dose ser repetida a cada 4 horas até a cianose ser debelada (De Castro et al., 2002; Malamed, 2004; Ferreira, 1999; Souza; Faria, 1991).

\section{Uso em gestantes}

Alguns aspectos devem ser observados quando da utilização de anestésicos locais em gestantes, dentre 
eles: técnica anestésica, quantidade da droga administrada, ausência/presença de vasoconstritor e os efeitos citotóxicos (Scavuzzi; Rocha, 1999).

$\mathrm{O}$ anestésico local pode afetar o feto de duas maneiras: diretamente (quando ocorrem altas concentrações na circulação fetal) e indiretamente (alterando o tônus muscular uterino ou deprimindo os sistemas cardiovascular e respiratório da mãe) (Oliveira, 1990).

A lidocaína é o anestésico mais apropriado para as gestantes, segundo a literatura pesquisada. Prilocaína e articaína não devem ser usadas por poderem levar à metahemoglobinemia, tanto na mãe quanto no feto (Malamed, 2004). A bupivacaína apresenta a maior cardiotoxicidade, maior penetrabilidade nas membranas do coração e maior resistência após eventual parada cardíaca. Em relação à mepivacaína, mais pesquisas devem ser realizadas já que seus riscos para o feto não são bem detalhados, portanto, seu uso é desaconselhado.

Quanto ao uso dos vasoconstrictores em gestantes, quando os benefícios superarem os riscos, os mesmos devem ser utilizados. Sem vasoconstrictor, o anestésico pode não ser eficaz, além de seu efeito passar mais rapidamente. A dor resultante pode levar o paciente ao estresse, fazendo com que haja liberação de catecolaminas endógenas em quantidades muito superiores àquelas contidas em tubetes anestésicos e, consequentemente, mais prejudiciais.

A felipressina deve ser evitada em pacientes grávidas por ser derivada da vasopressina e, teoricamente, ter capacidade de levar à contração uterina. Noradrenalina na concentração 1:25.000 e 1:30.000 não devem ser usadas, tendo em vista o grande número de complicações cardiovasculares e neurológicos causados por essa substância, sendo a concentração 1:50.000 a mais indicada (De Castro et al., 2002).

\section{Discussão}

A anestesia em Odontologia, apesar da segurança atualmente garantida pelos anestésicos, merece receber cuidados especiais na observação do estado de saúde do paciente, da correta seleção do agente anestésico, do manuseio e conservação dos tubetes anestésicos e, principalmente conhecimento adequado das técnicas anestésicas disponíveis e suas variações (Vieira; Gonçalves; Agra, 2000). O cirurgião-dentista deve estar sempre atento à dosagem do anestésico local utilizado e aos sinais e sintomas apresentados pelo paciente durante a realização da anestesia (Souza; Faria, 1991).

O grau de toxicidade de um anestésico vai depender do tipo de droga utilizada e do estado de saúde do paciente. A ocorrência de reação alérgica por anestésicos locais é bastante rara, mas o paciente pode relatar história pregressa de alguma reação interpretada como hipersensibilidade. Apenas 1\% das reações adversas que ocorrem em função de uma anestesia representam este tipo de reação (Vieira; Gonçalves; Agra, 2000).

A articaína juntamente com a prilocaína são os anestésicos que mais apresentam casos de parestesias mandibulares, sendo os únicos comercializados na concentração de 4\%. Também apresentam um risco 5x maior de causar neuropatias e podem levar também à metahemoglobinemia. A articaína consegue prover um efeito semelhante, estatisticamente, quando comparada aos outros anestésicos locais (Malamed, 2004; Mikesell et al., 2005).

A mepivacaína 3\% sem vasoconstritor é recomendada em pacientes nos quais não se indicam um vasocontritor e também em procedimentos que não requeiram anestesia pulpar de longa duração ou com grande profundidade (Malamed, 2004). Mendonça et al. (2003) verificaram que a mepivacaína foi a droga mais utilizada nos procedimentos realizados na Clínica Integrada da Universidade de Feira de Santana-BA. A maioria dos casos ficou dentro da dose máxima recomendada pela literatura e apenas dois pacientes apresentaram reações adversas.

Os agentes anestésicos novos (ropivacaína e levobupivacaína) podem ser considerados mais seguros que a bupivacaína, lembrando que essa segurança não é de 100\%. A ropivacaína apresenta menos reações neurotóxicas e cardiotóxicas que a levobupivacaína (Veering, 2003).

A American Dental Asssociation e a American Heart Association (1964) recomendam que ao paciente cardíaco se dê anestesia adequada ao ato cirúrgico que deverá ser realizado, pois os benefícios de uma boa anestesia superam em muito os riscos causados pela anestesia sem vasoconstritor, onde a absorção da base 
anestésica é rápida e nem sempre consegue-se um perfeito bloqueio nervoso (Tortamano; Armonia, 2001).

Em cardiopatas, consideram-se $54 \mu \mathrm{g}$ de adrenalina como dose limite por sessão ( 3 a 6 tubetes com concentrações respectivas de 1:100.000 e 1:200.000) (Ferreira, 1999). Uma vez associado o vasoconstrictor, a toxicidade sistêmica desaparece. Brkovic, Torodovic e Stojic (2005) sugeriram o uso da clonidina como alternativa mais segura para a adrenalina, uma vez que clonidina apresenta vantagens hemodinâmicas em relação à adrenalina.

A felipressina sendo uma análoga sintética da vasopressina torna-se um potente vasoconstrictor coronariano, mesmo sem apresentar efeitos sobre a musculatura miocárdica. Em indivíduos normais, isso não causaria problema nenhum, mas em pacientes que apresentam alguma deficiência da circulação coronariana, a literatura tem mostrado que, mesmo em pequenas doses, a vasopressina pode desencadear uma crise de angina neste paciente, com isquemia miocárdica. Poucos estudos foram realizados até o presente momento para avaliar a sua dose crítica, o que deixa ainda dúvidas sobre seus reais efeitos no coração. Pacientes portadores de diabetes mellitus, esclerose múltipla e que fazem tratamento quimioterápico, apresentam um risco mais alto de toxicidade sistêmica causada pelos anestésicos locais (Faria; Marzola, 2001; Tortamano; Armonia, 2001; Ferreira, 1999; Al-Nasser, 2002).

No tocante ao uso dos anestésicos locais em gestantes, conforme a literatura, a substância mais apropriada é a lidocaína a 2\% (De Castro et al., 2002). Todavia, segundo Malamed (2004) deve-se estar sempre atento à dose máxima de drogas administradas, quer sejam bases anestésicas, quer sejam vasoconstritores. Nos casos de tratamento invasivo não-emergencial deve-se postergar para após o nascimento da criança. Caso não seja possível adiar, deverá ser realizado, preferencialmente no segundo trimestre da gravidez (De Castro et al., 2002).

\section{Conclusão}

Com base no exposto, conclui-se que é importante ao cirurgião-dentista conhecimentos dos aspectos farmacológicos dos anestésicos locais, suas principais indicações e contra-indicações, além das possíveis reações locais e sistêmicas advindas do seu uso. Imprescindível se faz também a realização de uma completa anamnese e do manejo adequado da técnica escolhida, com a finalidade de oferecer ao paciente o melhor atendimento.

\section{REFERÊNCIAS}

1. AL-NASSER, B. Local toxicity of local anaesthetics - do experimental data apply to clinical manifestations? Anaesthesia, London, v. 57. n. 12, p. 1236-1237, Dec. 2002.

2. ARAÚJO, L. M. T.; AMARAL, J. L. G. Alergia à lidocaína. Relato de caso. Rev Brasileira de Anestesiologia, Rio de Janeiro, v. 54, n. 5, p. 672-676, set./out. 2004.

3. BERKUN, Y.; BEM-ZVI, A.; LEVY, Y.; GALILI, D.; SHALIT, M. Evaluation of adverse reactions to local anesthesic: experience with 236 patients. Annals of Allergy, Asthma and Immunology, St. Paul, v. 91, n. 4, p. 342-345, Oct. 2003.

4. BRKOVIC, B.; TORODOVIC, L.; STOJIC, D. Comparison of clonidine and epinephrine: anaesthesia for lower third molar surgery. Int J Oral Maxillofacial Surg, Copenhagen, v. 34, n. 4, p. 401-406, Jun. 2005.

5. DE CASTRO, F. C.; MENESES, M. T. V.; PORDEUS, I. A.; PAIVA, S. M. Tratamento odontológico no período da gravidez: enfoque para o uso de anestésicos locais. JBC, Curitiba, v. 6, n. 31, p. 62-67, jan./fev. 2002.

6. DEF. Dicionário de especialidades farmacêuticas 2004/05. 33. ed. Rio de Janeiro: Editora de Publicações Científicas. 2004.

7. FARIA, F. A. C.; MARZOLA, C. Farmacologia dos anestésicos locais - considerações gerais. BCI, Curitiba, v. 8. n. 29, p. 19-30, jan./mar. 2001.

8. FERREIRA, M.B. C. Anestésicos locais. In: WANNMACHER, L.; FERREIRA, M. B. C. Farmacologia clínica para dentistas. Rio de Janeiro: Guanabara-Koogan, 1999. Cap. 16. p.104-116.

9. MALAMED, S. F. Manual de anestesia local. 5. ed. São Paulo: Elsevier. 2004

10. MARIANO, R. C.; SANTANA, S. I.; COURA, G. S. Análise comparativa do efeito anestésico da lidocaína $2 \%$ e da prilocaína 3\%. BCI, Curitiba, v. 7, n. 27, p. 15-19, jul./set. 2000.

11. MENDONÇA, R. G.; FARIAS, J. G.; BARBOSA, A. S.; ALMEIDA, J. C.; GUIMARÃES, L. S. B. Verificação e análise da dose máxima anestésica local aplicada em 60 casos no ambulatório da clínica odontológica da Universidade Estadual de Feira de Santana. Rev CTBMF, Recife, v. 3, n. 4, p. 35-43, out./dez. 2003.

12. MIKESELL, P.; NUSSTEIN, J.; READER, A.; BECK, M.; WEAVER, J. A comparison of articaine and lidocaine of inferior alveolar nerve blocks. J Endond, Baltimore, v. 31, n. 4, p.265- 
270, Apr. 2005.

13. OLIVEIRA, M. F. M. Atendimento odontológico na gravidez. São Paulo: Santos, 1990.

14. PONZONI, D.; SANCHES, M. G.; OKAMOTO, T. Influência de solução anestésica local contendo mepivacaína no processo de reparo em feridas de extração dental: análise histológica em ratos. Rev ABO Nac, São Paulo, v. 11, n. 5, p. 287-292, out./ nov. 2003.

15. RANALI, J.; VOLPATO, M. C. Bupivacaína - anestésico local de longa duração: revisão sobre sua farmacologia e uso clínico em Odontologia. RBO, Rio de Janeiro, v. 47, n. 6, p. 3640, nov./dez. 1990.

16. SCAVUZZI, A. I. F.; ROCHA, M. C. B. S. Atenção odontológica na gravidez - uma revisão. Rev Fac Odontol Univ Fed
Bahia, Salvador, v. 18, p. 46-52, 1999.

17. SOUZA, J. A.; FARIA, M. T. P. Intoxicação sistêmica por anestesia local. Relato de um caso. RBO, Rio de Janeiro, v. 48, n. 3, p. 50-55, maio/jun. 1991.

18. TORTAMANO, N.; ARMONIA, P. L. Anestésicos locais. In: TORTAMANO, N.; ARMONIA, P.L. Guia terapêutico odontológico. 14. ed. São Paulo: Santos. 2001. Cap. 4, p. 30-41.

19. VEERING, B. Complications and local anaesthetic toxicity in regional anaesthesia. Curr Opin Anaesthesiol, Philadelphia, v. 16, n. 5, p. 455-459, Oct. 2003.

20. VIEIRA, G. F.; GONÇALVES, E. A. N.; AGRA, C. M. Anestesia odontológica: segurança e sucesso - parte 1. Rev Assoc Paul Cirur Dent, São Paulo, v. 54, n. 1, p. 42-45, jan./ fev. 2000. 